\title{
Occipital aneurysmal bone cyst rupture following head trauma: case report
}

\author{
Sarah T. Garber, MD, and Jay K. Riva-Cambrin, MD, MSc \\ Department of Neurosurgery, Primary Children's Medical Center, University of Utah, Salt Lake City, Utah
}

Aneurysmal bone cysts (ABCs) are benign, expansile, osteolytic lesions that represent $1 \%-2 \%$ of primary bone tumors. Cranial $A B C s$ are even more rare and represent $3 \%-6 \%$ of these unique lesions. The authors describe the case of a 3 -year-old girl who presented with an acute posterior fossa epidural hematoma after minor trauma. Imaging workup revealed a previously undiagnosed suboccipital $A B C$ that appeared to have ruptured as a result of her trauma, leading to a life-threatening hemorrhage. To the authors' knowledge, a ruptured $A B C$ has never before been presented in the pediatric literature. In this case report, the authors review the imaging findings, natural history, clinical course, and treatment of these rare lesions.

http://thejns.org/doi/abs/10.3171/2014.9.PEDS14224

KEY WORDS aneurysmal bone cyst; ruptured; trauma; pediatrics

$\Lambda$ NEURYSMAL bone cysts (ABCs) are expansile, vascular bony lesions. The typical location for an $\mathrm{ABC}$ is the metaphyses of the long bones, although approximately $20 \%$ present in the spine. ${ }^{7}$ Calvarial ABCs are very rare and represent only $3 \%-6 \%$ of these vascular bony lesions overall. ${ }^{5}$ Aneurysmal bone cysts are most common in patients younger than 20 years, and a slight female predominance has been reported in the literature. ${ }^{5}$ Several theories exist regarding the origin of $\mathrm{ABCs}$, with no one unifying theory present to explain the cause of these rare lesions. ${ }^{13}$ To our knowledge, a ruptured $\mathrm{ABC}$ as a result of head trauma has not been reported in the pediatric literature, and only one case report in the adult literature describes a similar phenomenon. ${ }^{1}$ We present the case of a 3-year-old girl who had a previously undiagnosed $\mathrm{ABC}$ at the foramen magnum that ruptured in the form of an acute compressive epidural hematoma (EDH) after head trauma. We review the presenting symptoms, imaging features, treatment, and natural history of these rare lesions.

\section{Case Report}

History and Presentation

A previously healthy 3-year-old girl was brought to our institution after falling off the monkey bars equipment at a playground and striking the back of her head. The patient vomited twice on the scene, and she was lethargic with a Glasgow Coma Scale score of 10 on presentation, with reactive pupils and symmetrical movements to stimulation. A CT scan obtained without contrast revealed a leftsided posterior fossa EDH overlying the transverse sinus. There was effacement of the fourth ventricle and dilation of the temporal horns (Fig. 1). Irregular bone loss around the posterior margin of the foramen magnum and occipital bone, with an associated soft-tissue mass, was also noted (Fig. 2). The family was unaware of this lesion prior to this event. The CT scan was reviewed by a neuroradiologist and thought to represent an $\mathrm{ABC}$, and because of the patient's neurological compromise, an emergency MRI study was not obtained. Because of the often bloody nature of these lesions, the operating room staff was notified of blood typing and screening results before the patient was taken to the operating room, and unmatched $\mathrm{O}$-negative packed red blood cells were made available in case of urgent need.

\section{Operation}

The patient underwent an emergency posterior fossa craniectomy with gross-total resection (GTR) of the bony lesion and evacuation of the EDH. Intraoperatively, the mass had eroded the margins of the foramen magnum and was removed en bloc, although there was brisk bleeding

ABBREVIATIONS ABC = aneurysmal bone cyst; $\mathrm{EDH}=$ epidural hematoma; $\mathrm{GTR}=$ gross-total resection.

SUBMITTED April 30, 2014. ACCEPTED September 12, 2014.

INCLUDE WHEN CITING Published online January 2, 2015; DOI: 10.3171/2014.9.PEDS14224.

DISCLOSURE The authors report no conflict of interest concerning the materials or methods used in this study or the findings specified in this paper. 

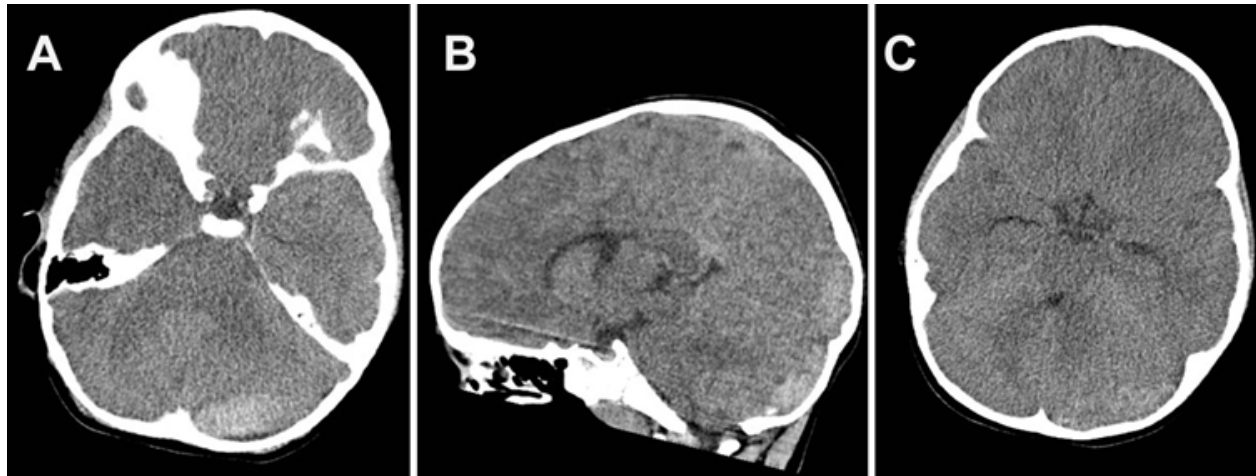

FIG. 1. Axial (A and C) and sagittal (B) head CT scans obtained without contrast showing a left posterior fossa EDH extending superior and inferior to the transverse sinus, causing effacement of the fourth ventricle and dilation of the temporal horns (C).

necessitating blood transfusion. Once the EDH was evacuated, we closely inspected the transverse sinus for tears; no injury to the sinus was found as an alternative source for the EDH. All bleeding from the edges of the craniotomy was stopped with bone wax to help prevent air embolism. An intraoperative ultrasound confirmed that there was no subdural or intraparenchymal hemorrhage, nor was there obvious thrombosis within the venous sinus.

\section{Postoperative Course}

Postoperatively, the patient was extubated and taken to the pediatric ICU in stable condition. An MRI study of the brain and cervical spine was performed on postoperative Day 1 , which was consistent with a GTR of the mass and decreased effacement of the fourth ventricle (Fig. 3). The
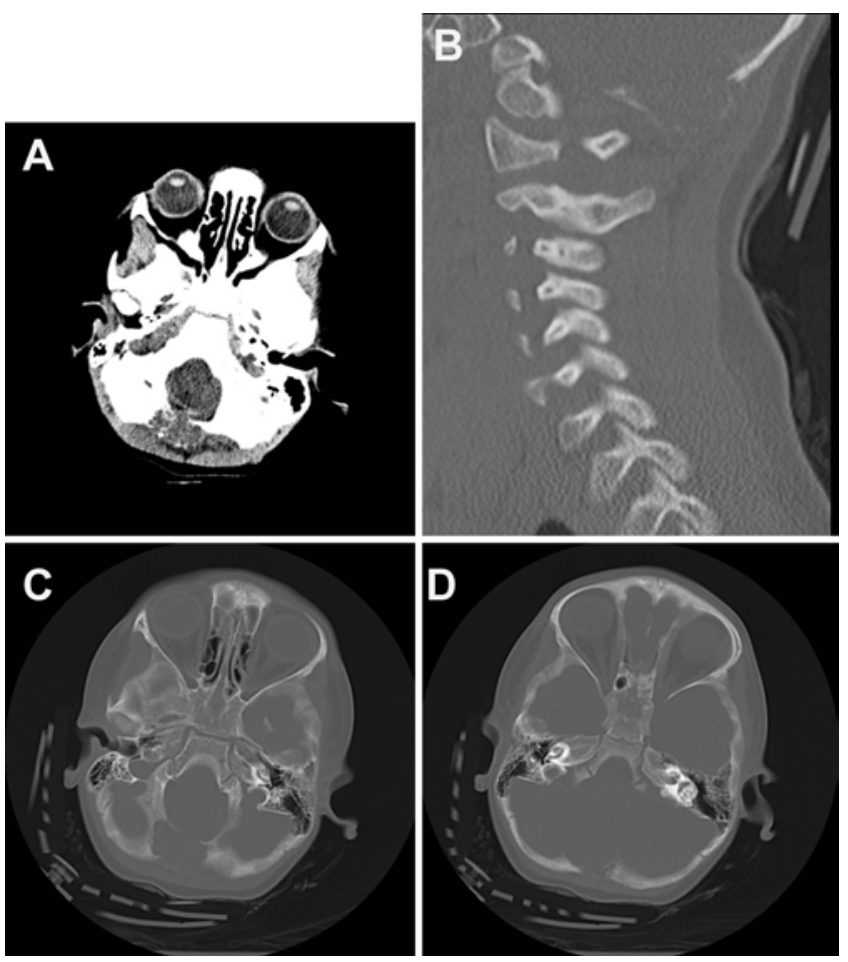

FIG. 2. Axial head CT (A) and sagittal CT of the cervical spine (B), both obtained without contrast, showing a mass at the level of the foramen magnum with erosion of the surrounding bone. Bone windows ( $C$ and $D$ ) show the irregular margins of this lesion. patient was discharged home on postoperative Day 2 neurologically intact. On pathological examination, the lesion showed large cystic spaces filled with blood and separated by fibrous septa, alternating with more solid areas. There were also clusters of multinucleated giant cells with loose, spindly stroma, but no malignant osteoid and no atypia (Fig. 4). The final pathological diagnosis was a ruptured $\mathrm{ABC}$. The patient has been seen at 1- and 6-month follow-up and remains neurologically intact. Follow-up CT scans and MRI studies confirm GTR and no recurrence at 6 months.

\section{Discussion}

\section{Origin of $A B C s$}

Several theories exist to explain the origin of ABCs. One theory hypothesizes that an inciting trauma induces a local circulatory disturbance that leads to the development of an ABC. ${ }^{13}$ Another theory is that ABCs are actually neoplasms with unique cytogenetic abnormalities. ${ }^{7}$ Approximately $50 \%$ of patients with these lesions show an abnormality on chromosome $16 \mathrm{q}$ or $17 \mathrm{p}$. This is typically a chromosomal translocation that causes upregulation of ubiquitin-specific proteases, leading to increased cell-cell adhesion. ${ }^{7} \mathrm{~A}$ third theory posits that $\mathrm{ABCs}$ arise as a result of an underlying neoplasm that induces a vascular process such as a venous obstruction or an arteriovenous fistula. ${ }^{9}$ In fact, up to $30 \%$ of $\mathrm{ABCs}$ are associated with underly-
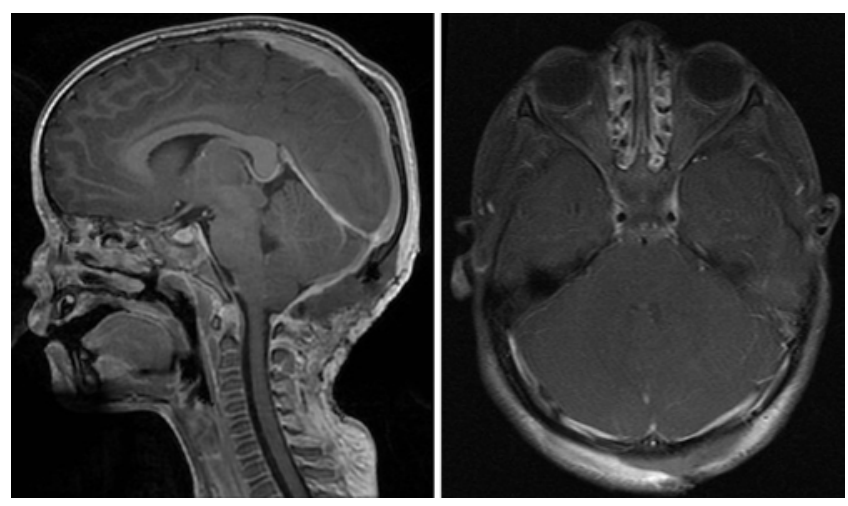

FIG. 3. Sagittal (left) and axial (right) contrast-weighted MRI studies showing resolution of the mass effect on the fourth ventricle and a patent transverse sinus with GTR of the mass. 

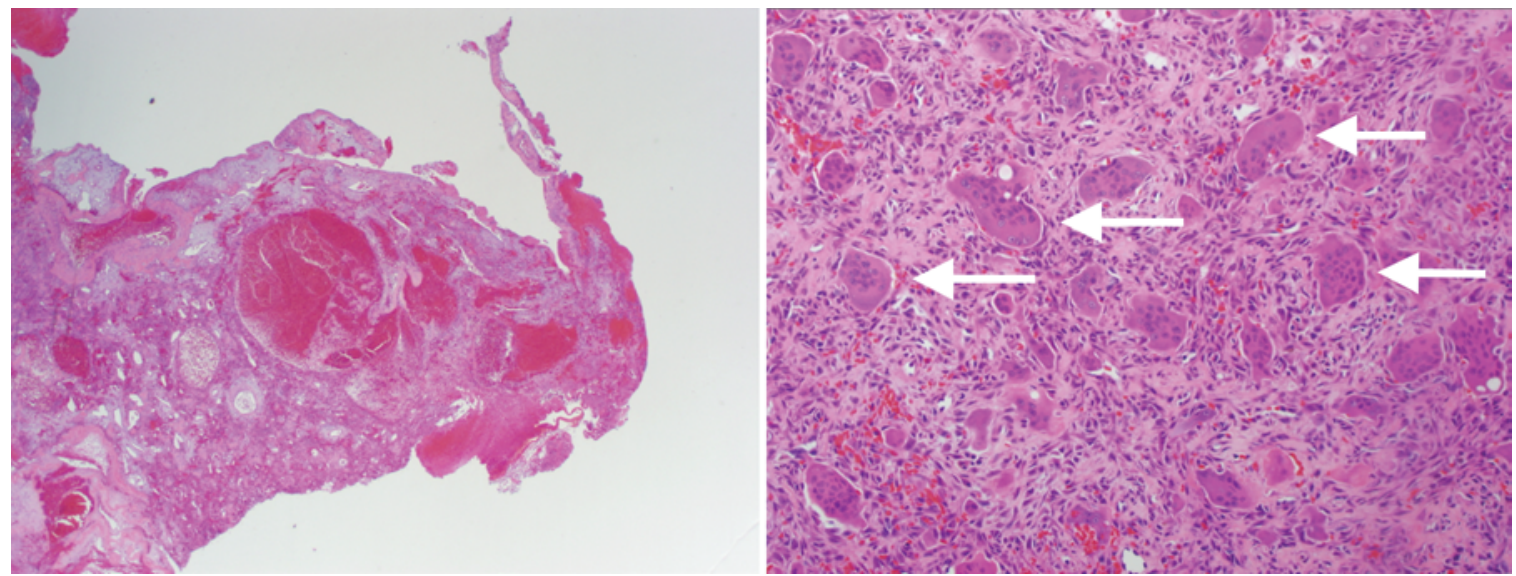

FIG. 4. Photomicrographs of specimens taken from the lesion demonstrating large cystic spaces filled with blood separated by a fibrous stroma (left) and multinucleated giant cells without cytological atypia (arrows) (right). H \& E, original magnification $\times 10$ (left) and $\times 100$ (right). Figure is available in color online only.

ing tumors such as giant cell tumors, chondroblastoma, osteoblastoma, osteosarcoma, and fibrous dysplasia. ${ }^{14} \mathrm{~A}$ hormonal influence on the growth of these tumors has also been proposed, because they do tend to have a slight female predominance and have been shown to increase in size during pregnancy; ${ }^{10}$ however, these lesions have displayed negative results on immunohistochemical investigation for estrogen or progesterone receptors. Given that our patient had no history of previous head trauma and no underlying neoplasm was found on pathological or radiological examination, it is possible that the second theory may be most applicable in this case.

\section{Imaging Findings}

On CT scans, patients with ABCs of the cranium may demonstrate thinning of the cortical bone surrounding the lesion, resulting in focal cortical destruction. ${ }^{2}$ The cysts present in an $\mathrm{ABC}$ are separated by septae of varying thicknesses, and fluid-fluid levels are often seen because of hemorrhage inside the cysts. The intensity of an $\mathrm{ABC}$ on MRI varies depending on the stage of hematoma degradation. These cysts can also demonstrate peripheral enhancement with the administration of intravenous contrast.

\section{Presentation and Treatment}

Typically, ABCs present in patients during the 1st or 2nd decade of life. The most common presenting symptom is pain at the site of the ABC. ${ }^{9}$ When an $\mathrm{ABC}$ presents in the spine, epidural compression and cortical erosion can lead to scoliosis, fractures, or neurological symptoms from root or cord compression. ${ }^{2}$ To our knowledge, the patient described in this case report did not complain of any posterior neck pain or headaches localizing to the site of her $\mathrm{ABC}$ prior to her minor fall.

Hemorrhagic skull lesions in the pediatric population are extremely rare. Both intraosseous hemangiomas and $\mathrm{ABCs}$ can present as benign, enlarging skull masses, although typically intraosseous hemangiomas are painless, whereas $\mathrm{ABCs}$ are painful to the touch. ${ }^{3}$ Osteoid osteo- mas, which represent $10 \%-12 \%$ of osseous tumors in the pediatric population and are distinguished from osteoblastoma by size $(<1.5 \mathrm{~cm})$, are also composed of highly vascular connective tissue, although these lesions are more typically found in the posterior elements of the cervical spine. ${ }^{6}$ As far as more malignant skull lesions are concerned, osteosarcomas of the skull base can present with hemorrhage, ${ }^{11}$ although these are more common in older patients (3rd-4th decade of life), whereas osteosarcoma of the long bones tends to present in adolescents. ${ }^{4}$ The rate of hemorrhage of these lesions is largely unknown.

The treatment of an ABC consists of GTR whenever possible. There is a reported 3\% rate of malignant degeneration into osteosarcoma, and incomplete resection is associated with a recurrence rate of $71 \% .^{9}$ When treating an $\mathrm{ABC}$ of the spinal column, resection may be accompanied by spinal fusion to address destabilization of the spine after lesion removal; however, this is not relevant to our suboccipital case. ${ }^{8}$ Preoperative embolization is also an option because of the vascular nature of these lesions and their propensity for intraoperative bleeding. Because of the rare possibility that an $\mathrm{ABC}$ may degenerate into a more malignant entity such as an osteosarcoma, radiation alone is no longer recommended as a sole treatment for these lesions. ${ }^{8,12}$ Partial excision with adjuvant radiation may be required for lesions that are not amenable to GTR, such as $\mathrm{ABC}$ located in the orbit, paranasal sinuses, or petrous temporal bone.

Other modalities that have been used with varying success for the treatment of ABCs include CT-guided percutaneous lesional injection of calcitonin and methylprednisolone..$^{12}$ Calcitonin inhibits osteoclastic activity and promotes trabecular bone formation. Methylprednisolone has an antiangiogenesis effect. Additionally, the use of sclerosants such as alcohol has been tried; these act by direct damage to the endothelial lining, triggering a coagulation cascade and thrombotic occlusion of blood vessels. ${ }^{12}$ Because our patient displayed no residual lesion on postoperative imaging, the current plan is observation without adjuvant therapy. Another CT scan will be performed at an 18-month follow-up visit. 


\section{Conclusions}

Aneurysmal bone cysts are rare lesions that typically present in the long bones or the spine in young patients. Several theories exist regarding the origin of these lesions, and treatment consists of GTR whenever possible. The presence of an $\mathrm{ABC}$ in the cranium is extremely rare in both the pediatric and the adult populations, and a ruptured ABC after head trauma has not been reported previously. We do not know whether these lesions are capable of spontaneous rupture, although there is an association with the development of $\mathrm{ABC}$ at the site of a prior trauma. Knowledge of the possibility that an $\mathrm{ABC}$ may rupture is useful for early recognition and diagnosis of this entity and prompt surgical treatment.

\section{Acknowledgment}

We thank Kristin Kraus, MSc, for her help with the preparation of this manuscript.

\section{References}

1. Arnaldsson OS, Ragnarsson T: [Aneurysmal bone cyst, following a skull trauma. A case report.] Laeknabladid 81:799-802, 1995 (Icelandic)

2. Boriani S, De Iure F, Campanacci L, Gasbarrini A, Bandiera S, Biagini R, et al: Aneurysmal bone cyst of the mobile spine: report on 41 cases. Spine (Phila Pa 1976) 26:27-35, 2001

3. Cosar M, Eser O, Aslan A, Korkmaz S, Boyaci G, Aktepe F: Intradiploic cavernous hemangioma of the skull in a child: a case report. Childs Nerv Syst 24:975-977, 2008

4. Eftekhari F: Imaging assessment of osteosarcoma in childhood and adolescence: diagnosis, staging, and evaluating response to chemotherapy. Cancer Treat Res 152:33-62, 2009

5. Gan YC, Hockley AD: Aneurysmal bone cysts of the cranium in children. Report of three cases and brief review of the literature. J Neurosurg 106 (5 Suppl):401-406, 2007

6. Kan P, Schmidt MH: Osteoid osteoma and osteoblastoma of the spine. Neurosurg Clin N Am 19:65-70, 2008
7. Lau AW, Pringle LM, Quick L, Riquelme DN, Ye Y, Oliveira AM, et al: TRE17/ubiquitin-specific protease 6 (USP6) oncogene translocated in aneurysmal bone cyst blocks osteoblastic maturation via an autocrine mechanism involving bone morphogenetic protein dysregulation. J Biol Chem 285:37111-37120, 2010

8. Liu JK, Brockmeyer DL, Dailey AT, Schmidt MH: Surgical management of aneurysmal bone cysts of the spine. Neurosurg Focus 15(5):E4, 2003

9. Mankin HJ, Hornicek FJ, Ortiz-Cruz E, Villafuerte J, Gebhardt MC: Aneurysmal bone cyst: a review of 150 patients. $\mathbf{J}$ Clin Oncol 23:6756-6762, 2005

10. Mintz MC, Dalinka MK, Schmidt R: Aneurysmal bone cyst arising in fibrous dysplasia during pregnancy. Radiology 165:549-550, 1987

11. Niazi TN, Forester C, Afify Z, Riva-Cambrin J: Osteosarcoma presenting as hemorrhagic cerebellar metastasis. Childs Nerv Syst 25:1643-1647, 2009

12. Papagelopoulos PJ, Choudhury SN, Frassica FJ, Bond JR, Unni KK, Sim FH: Treatment of aneurysmal bone cysts of the pelvis and sacrum. J Bone Joint Surg Am 83-A:16741681,2001

13. Rapp TB, Ward JP, Alaia MJ: Aneurysmal bone cyst. J Am Acad Orthop Surg 20:233-241, 2012

14. Segall L, Cohen-Kerem R, Ngan BY, Forte V: Aneurysmal bone cysts of the head and neck in pediatric patients: a case series. Int J Pediatr Otorhinolaryngol 72:977-983, 2008

\section{Author Contributions}

Conception and design: both authors. Acquisition of data: both authors. Drafting the article: both authors. Critically revising the article: both authors. Reviewed submitted version of manuscript: both authors. Approved the final version of the manuscript on behalf of both authors: Riva-Cambrin.

\section{Correspondence}

Jay K. Riva-Cambrin, Department of Neurosurgery, Primary Children's Medical Center, 100 Mario Capecchi Dr., Salt Lake City, UT 84113. email: jay.riva-cambrin@hsc.utah.edu. 